\title{
A Real-Time SNR Estimator for D-MPSK over Frequency-Flat Slow Fading AWGN Channels
}

\author{
Yair Linn \\ University of British Columbia \\ Vancouver, BC, Canada \\ e-mail: ylinn@ece.ubc.ca
}

\begin{abstract}
We present a channel SNR (Signal-to-Noise Ratio) estimator for D-MPSK (Differential M-ary Phase Shift Keying) in AWGN (Additive White Gaussian Noise). The estimator is shown to have the following advantages: (1) the estimator has a compact fixed-point hardware implementation; (2) it requires only 1 sample/symbol; (3) accurate estimates can be generated in real-time; (4) the estimator is resistant to imperfections in the AGC (Automatic Gain Control) circuit. We investigate the proposed estimator theoretically and through simulations. General formulas are developed for SNR estimation in the presence of frequency-flat slow fading, and specific results are presented for Nakagami-m fading. The proposed estimator is then compared to other SNR estimators, and it is shown that the proposed method requires less hardware resources while at the same time providing superior performance. Finally, we briefly consider application of the proposed SNR estimator to SNR estimation in coherent M-PSK receivers when carrier synchronization has not been achieved.
\end{abstract}

\section{INTRODUCTION}

One of the most important signal metrics in any receiver's operation is an estimate of the received signal's $E_{S} / N_{0}$ ratio or SNR (these terms are used here interchangeably). Two examples are systems that employ diversity reception [1 Sec. 14.4] (for which SNR estimates are used to assign relative weights to the data obtained from the various receivers), and adaptive schemes where the data and/or coding rates are altered according to the SNR. Moreover, some error correction decoders can make use of an SNR estimate to increase their coding gain (e.g. turbo codes [2]).

In this paper we present a robust real-time SNR estimator for DMPSK. This estimator is a modification of the Linn-Peleg estimator ([3], [4]) for coherent M-PSK, and, as such, it retains the advantages which were observed for the latter. Specifically, the estimator is shown to have excellent performance and an exceptionally compact hardware implementation which is quite suitable for implementation within FPGAs (Field Programmable Gate Arrays) or ASICs (Application Specific Integrated Circuits). The resilience to AGC circuit imperfections that was observed for the Linn-Peleg estimator ([3], [4]) is also observed for the current structure.

\section{SYSTEM MODEL}

Signal and receiver characteristics are assumed identical to those in [3 Sec. II] and [4 Sec. II], except that here (since we use D-MPSK) we do not have a carrier recovery PLL. The reader is strongly urged to take a thorough look at [3] and [4], since notations and results from both papers will be used extensively. The baseband PSK signal is $m(t) \triangleq \sum_{n=-\infty}^{\infty} a_{n} p(t-n T)$, with $p(t)$ being the pulse shape and $\quad a_{n}=\exp \left(j \phi_{n}\right)$, $\phi_{n}=2 \pi \cdot m_{n} / M$, with $m_{n} \in\{0,1, \ldots, M-1\}$. The modulated signal is $s_{m}(t) \triangleq \operatorname{Re}\left[m(t) \exp \left(j \omega_{i} t+j \theta_{i}\right)\right]$. A simplified diagram of the front-end of the receiver under discussion is shown in Fig. 1. The matched filter response is $p(-t)$. From [3 Sec. II] we have $I(n)=K\left(2 E_{S} \cdot \cos \left(-\Delta \omega \cdot n T+\theta_{e}+\phi_{n}\right)+n_{I}(n T)\right) \quad$ and $Q(n)=K\left(2 E_{S} \cdot \sin \left(-\Delta \omega \cdot n T+\theta_{e}+\phi_{n}\right)+n_{Q}(n T)\right), \quad$ with $\theta_{e} \triangleq \theta_{i}-\theta_{o}$ and $n_{I}(n T), n_{Q}(n T) \sim N\left(0,2 N_{0} E_{S}\right) . K$ is the equivalent (AGC-controlled) $I-Q$ arm gain [3 Sec. III] (see [5 App. A] for a thorough discussion of the AGC and the parameter $K$ ). The phase of the complex symbol $r_{n} \triangleq I(n)+j \cdot Q(n) \quad$ is $\varphi_{n} \triangleq \tan ^{-1}(Q(n) / I(n)) . \quad$ We then have $r_{n}=\left|r_{n}\right| \exp \left(j \varphi_{n}\right)$. Here (unlike in [3], [4]), we do not assume $\Delta \omega=0$, but rather $|\Delta \omega| \ll 2 \pi /(M \cdot T)$, which is the standard assumption that is made in D-MPSK receivers (e.g. [6 Sec. 10.19]). We assume that our signal is subject to frequency-flat (=frequencynonselective) slow fading (see for example [1 Sec. 14.3]).

\section{Motivation AND Estimator STRUCTURE}

\section{A. Motivation}

Detection of D-MPSK signals is often facilitated by first generating a pseudo-coherently demodulated M-PSK signal $u_{n} \triangleq r_{n} r_{n-1}^{*}$ and then applying M-PSK decision regions upon $u_{n}$. The motivation here is similar, but we add a twist: the idea is to use the Linn-Peleg M-PSK SNR estimator ([3], [4]) upon a normalized pseudo-coherently demodulated M-PSK signal $v_{n} \triangleq \frac{r_{n} r_{n-1}^{*}}{\left|r_{n}\right|\left|r_{n-1}\right|}$. As we shall see, using $v_{n}$ instead of $u_{n}$ yields a simpler hardware implementation.

\section{B. Estimator Structure and Operation Principle}

We define $x_{M, n}^{D} \triangleq \operatorname{Re}\left[\left(v_{n}\right)^{M}\right] /\left|v_{n}\right|^{M} \quad$ (Note: to avoid confusion with [3] and [4], throughout this paper we use superscript " $D$ " in variables pertaining to D-MPSK structures). The Linn-Peleg detector ([3], [4]) applied to $v_{n}$ is defined as:

$$
\hat{l}_{M, N}^{D} \triangleq \frac{1}{2 N} \sum_{n=-N+1}^{N} x_{M, n}^{D}=\frac{1}{2 N} \sum_{n=-N+1}^{N} \operatorname{Re}\left[\left(v_{n}\right)^{M}\right] /\left|v_{n}\right|^{M}
$$

Here we do not use $\hat{l}_{M, N}^{D}$ as a lock detector (since there is no carrier PLL) but rather only as an SNR estimator. Note that $\left|v_{n}\right|=1$ for all 
$n$, so theoretically we could have defined $\hat{l}_{M, N}^{D} \triangleq \frac{1}{2 N} \sum_{n=-N+1}^{N} \operatorname{Re}\left[\left(v_{n}\right)^{M}\right]$. However, when quantization effects are taken into account we see that $\left|v_{n}\right|=1$ does not always hold, and then $\quad \hat{l}_{M, N}^{D} \triangleq \frac{1}{2 N} \sum_{n=-N+1}^{N} \operatorname{Re}\left[\left(v_{n}\right)^{M}\right] /\left|v_{n}\right|^{M} \quad$ has distinct implementation and performance advantages (the same as outlined in [3 Sec. III-B]). In this paper we present a general method for SNR estimation in the presence of fading. We assume that the channel is underspread, i.e. that the fading is frequency-flat and slow ("slow" meaning that $T_{\mathrm{COH}}>T$, where $T_{\mathrm{COH}}$ is the channel coherence time [1 Sec. 14.1.1]). We use the notation $\chi$ to refer to the instantaneous SNR, and the notation $\bar{\chi}$ to denote the average SNR (i.e., $\left.\bar{\chi} \triangleq E\left[E_{S} / N_{0}\right]\right)$. The conditional pdf (probability density function) of the SNR due to fading will be denoted as $p_{F}(\chi \mid \bar{\chi}) \triangleq p\left(E_{S} / N_{0}=\chi \mid E\left[E_{S} / N_{0}\right]=\bar{\chi}\right)$. For example, from $[7$ Table 2] we have for Nakagami-m fading $p_{F}(\chi \mid \bar{\chi})=\frac{m^{m} \chi^{m-1}}{\bar{\chi}^{m} \Gamma(m)} \exp \left(\frac{-m \chi}{\bar{\chi}}\right)$.

We differentiate between two cases: (a) $2 N T \ll T_{\mathrm{COH}}$ and (b) $2 N T \gg T_{\mathrm{COH}}$. For case (a), during the averaging over $2 N T$ symbol intervals that is done in eq. (1) the channel SNR will not have changed much; hence SNR estimation from $\hat{l}_{M, N}^{D}$ will yield an estimate of the instantaneous SNR ratio $\chi$. For case (b), since $2 N T$ is much larger than the channel coherence time, SNR estimation from $\hat{l}_{M, N}^{D}$ will yield an estimate of the average SNR ratio $\bar{\chi}$.

Before continuing, it is important to note that the case $2 N \cdot T \simeq T_{\mathrm{COH}}$ (where " $\simeq$ " means the same order of magnitude) is undesirable since in that case the SNR distribution during the estimator computation interval cannot be predicted (it would be impossible to know which part of the fading pdf we experience during the computation interval). Thankfully, $2 N \cdot T \simeq T_{\mathrm{COH}}$ can always be averted by choosing a large enough $N$, which ensures that $2 N \cdot T \gg T_{C O H}$ (case (b)). In general, though, we would ideally like to produce SNR estimates which are instantly available and can fed in real-time to the decoder, equalizer, or other receiver components which could make good use of them. Hence, ideally, we would be served by perfect knowledge of the instantaneous SNR ratio $\chi$ (which, if desired, could be averaged over time in order to produce an estimate of $\bar{\chi}$ ). However, estimation of $\chi$ is not always possible, due to the fact that $T_{\mathrm{COH}}$ may be too short as compared to the estimation period $2 N T$ which is necessary in order to achieve an acceptable accuracy in the SNR estimation (see Secs. V-VII). Nonetheless, if $2 N T \gg T_{\mathrm{COH}}$, timely knowledge of the average SNR $\bar{\chi}$ is often sufficient to facilitate substantial performance gains.

SNR estimation is achieved following a procedure analogous to that in [4 eq. (9)], i.e. we estimate the SNR via the following:

Case (a) ( $2 N T \ll T_{\mathrm{COH}}$ ): instantaneous SNR is estimated via $\gamma_{d B}^{D}=10 \cdot \log _{10}\left(\left(f_{M}^{D}\right)^{-1}\left(\hat{l}_{M, N}^{D}\right)\right)$

where $f_{M}^{D}(\chi) \triangleq E\left[\hat{l}_{M, N}^{D} \mid E_{S} / N_{0}=\chi\right]$.

Case (b) ( $\left.2 N T \gg T_{C O H}\right):$ the average SNR is estimated via

$\bar{\gamma}_{d B}^{D}=10 \cdot \log _{10}\left(\left(\bar{f}_{M}^{D}\right)^{-1}\left(\hat{l}_{M, N}^{D}\right)\right)$

where $\bar{f}_{M}^{D}(\bar{\chi}) \triangleq E\left[\hat{l}_{M, N}^{D} \mid E\left[E_{S} / N_{0}\right]=\bar{\chi}\right]$.

\section{Hardware Implementation}

A fixed-point (2's complement) hardware implementation of the estimator is shown in Fig. 2. The LUTs (Lookup Tables) require

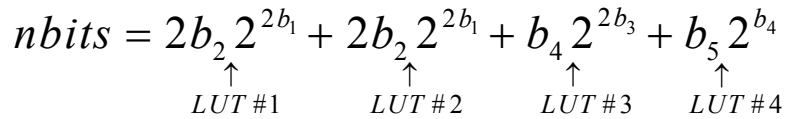

bits. See [3 Secs. III-B, III-C], and [4 Sec. III-B] for discussions applicable to LUT \#3 and LUT \#4, as well as discussion of why $2 N$ should be a power of 2 . The use of $v_{n}$ rather than $u_{n}$ significantly reduces the hardware resources needed to compute $\hat{l}_{M, N}^{D}$; this is because the normalized constellation has less dynamic range (it is $[-1,1])$ so this reduces $b_{2}$ and $b_{3}$ required to achieve an acceptable degradation due to quantization. Finally, it is noted that the Integrate and Dump (IAD) module is very simple to implement: it is essentially a register with an adder in the feedback path and some control logic.

\section{CONDitional Distribution of $\hat{l}_{M, N}^{D}$}

In this section we shall derive the conditional probability distribution of $\hat{l}_{M, N}^{D}$. These stochastic properties will then be used to develop the SNR estimation method in Section V. Without loss of generality (see [3 Sec. IV]) we assume $\forall n, \phi_{n}=0$, whereupon

$\varphi_{n}=\tan ^{-1}\left(\frac{\sin \left(-\Delta \omega \cdot n T+\theta_{e}\right)+n_{Q}(n T) /\left(2 E_{S}\right)}{\cos \left(-\Delta \omega \cdot n T+\theta_{e}\right)+n_{I}(n T) /\left(2 E_{S}\right)}\right)$

Let us define (similar to [3 eq. (32)]):

$\Delta \phi_{n} \triangleq \varphi_{n}-\left(-\Delta \omega n T+\theta_{e}\right)$

Since we assumed $\forall n, \phi_{n}=0$ then the physical meaning of $\Delta \phi_{n}$ is clear: it is the phase error in the received symbol that can be attributed to $n_{I}(n T)$ and $n_{Q}(n T)$ (to see this, substitute $n_{I}(n T)=n_{Q}(n T)=0$ in the expressions for $I(n)$ and $Q(n)$, and then $\varphi_{n}=\tan ^{-1}(Q(n) / I(n))=-\Delta \omega n T+\theta_{e}$ $\left.\Rightarrow \Delta \phi_{n}=\varphi_{n}-\left(-\Delta \omega n T+\theta_{e}\right)=0\right)$. Since (5) has the same form as [3 eq. (31)] with $\theta_{e}$ replaced by $-\Delta \omega n T+\theta_{e}$, we have that $\Delta \phi_{n}$ as defined in (6) is distributed the same as $\Delta \phi_{n}$ as defined in [3 eq. (32)], namely at $E_{S} / N_{0}=\chi$ it has a Rician phase pdf given by [3 eq. (19)]: 


$$
\begin{aligned}
& p_{C}(\Delta \phi \mid \chi) \triangleq p\left(\Delta \phi_{n}=\Delta \phi \mid E_{S} / N_{0}=\chi\right)=\frac{\exp (-\chi)}{2 \pi} \\
& \times\left[1+\sqrt{2 \chi} \cos (\Delta \phi) \exp \left(\chi \cdot \cos ^{2}(\Delta \phi)\right) \cdot \int_{-\infty}^{\cos (\Delta \phi) \sqrt{2 \chi}} e^{-y^{2} / 2} d y\right]
\end{aligned}
$$

where $-\pi \leq \Delta \phi \leq \pi$. Now, let us investigate $v_{n}$. Trivial substitutions

show

$v_{n}=\frac{\left|r_{n}\right| \exp \left(j \varphi_{n}\right)\left|r_{n-1}\right| \exp \left(-j \varphi_{n-1}\right)}{\left|r_{n}\right|\left|r_{n-1}\right|}=\exp \left(j \varphi_{n}^{D}\right)$

where

$\varphi_{n}^{D} \triangleq \varphi_{n}-\varphi_{n-1}$. Observe that $\varphi_{n}^{D} \in[-2 \pi, 2 \pi]$, though the true phase is $\varphi_{n}^{D} \bmod _{2 \pi} \in[-\pi, \pi]$. We could have indeed performed the modulo operation and confined the range of $\varphi_{n}^{D}$ to $[-\pi, \pi]$; this is, in fact, the approach undertaken in [8] and [9]. In contrast, we maintain the pretense $\varphi_{n}^{D} \in[-2 \pi, 2 \pi]$ because it simplifies the analysis. However, note that since $v_{n}=\exp \left(j \varphi_{n}^{D}\right)=\exp \left(j\left(\varphi_{n}^{D} \bmod _{2 \pi}\right)\right)$ this choice has no bearing upon the results. From (6) we then have:

$\varphi_{n}^{D}=\varphi_{n}-\varphi_{n-1}$

$=\Delta \phi_{n}+\left(-\Delta \omega \cdot n T+\theta_{e}\right)-\left(\Delta \phi_{n-1}+\left(-\Delta \omega \cdot(n-1) T+\theta_{e}\right)\right)$

$=\Delta \phi_{n}-\Delta \phi_{n-1}-\Delta \omega T$

Let us define $\Delta \phi_{n}^{D} \triangleq \Delta \phi_{n}-\Delta \phi_{n-1} ;$ note that since $\Delta \phi_{n}, \Delta \phi_{n-1} \in[-\pi, \pi]$ then $\Delta \phi_{n}^{D} \in[-2 \pi, 2 \pi]$. The pdf of $\Delta \phi_{n}^{D}$ is easily found since it is a convolution of the distributions of $\Delta \phi_{n}$ and $\left(-\Delta \phi_{n-1}\right)$, namely (for $-2 \pi \leq \Delta \phi^{D} \leq 2 \pi$ )

$$
\begin{aligned}
& p_{D}\left(\Delta \phi^{D} \mid \chi\right) \triangleq p\left(\Delta \phi_{n}^{D}=\Delta \phi^{D} \mid E_{S} / N_{0}=\chi\right) \\
& =\int_{-\pi}^{\pi} p_{C}(\tau \mid \chi) p_{C}\left(\tau-\Delta \phi^{D} \mid \chi\right) d \tau
\end{aligned}
$$

which is straightforward to evaluate numerically. Furthermore, a simple expression for the distribution at high SNR is easily obtained: from [3 eq. (22)] $\forall n, \Delta \phi_{n} \stackrel{\chi \rightarrow \infty}{\sim} N(0,1 /(2 \chi))$; now, since $\Delta \phi_{n}^{D}=\Delta \phi_{n}-\Delta \phi_{n-1} \quad$ and since $\Delta \phi_{n} \quad$ and $\Delta \phi_{n-1}$ are independent ([3 Sec. III $], \quad\left[\begin{array}{lll}10 & \text { Sec. 7.1.1 }\end{array}\right)$ we have $\Delta \phi_{n} \stackrel{\chi \chi \infty}{\sim} N(0,1 / \chi)$, i.e.

$p_{D}\left(\Delta \phi^{D} \mid \chi\right) \stackrel{\chi \rightarrow \infty}{\approx} \sqrt{\chi / 2 \pi} \cdot \exp \left(-0.5 \cdot \chi \cdot\left(\Delta \phi^{D}\right)^{2}\right)$

We now have the tools to investigate the distribution of $\hat{l}_{M, N}^{D}$.

\section{A. Conditional Expectation of $\hat{l}_{M, N}^{D}$ given $\chi$ for $2 N T \ll T_{C O H}$}

For $2 N T \ll T_{C O H}$, we can assume that the $E_{S} / N_{0}$ ratio is constant over the estimation interval and is equal to the instantaneous SNR $\chi$, i.e. this is equivalent to a case where no fading is present during the estimator's computation process. Hence (omitting some of the intermediate derivations, due to space constraints):

$$
\begin{aligned}
& f_{M}^{D}(\chi) \triangleq E\left[\hat{l}_{M, N}^{D} \mid \frac{E_{S}}{N_{0}}=\chi\right]=E\left[x_{M, n}^{D} \mid \frac{E_{S}}{N_{0}}=\chi\right] \\
& =E\left[\cos \left(M \Delta \phi_{n}^{D}\right) \mid \frac{E_{S}}{N_{0}}=\chi\right] \cos (M \Delta \omega T) \\
& =\left(\int_{-2 \pi}^{2 \pi} \cos \left(M \Delta \phi^{D}\right) p_{D}\left(\Delta \phi^{D} \mid \chi\right) d\left(\Delta \phi^{D}\right)\right) \cos (M \Delta \omega T)
\end{aligned}
$$

At high SNR we use (10) to obtain a useful approximation (using $\int_{0}^{\infty} e^{-a x^{2}} \cos (b x) d x=\frac{1}{2} \sqrt{\frac{\pi}{a}} e^{-b^{2} /(4 a)}$ [11 eq. (15.73)]): $f_{M}^{D}(\chi)$

$$
\begin{aligned}
& \stackrel{\chi \rightarrow \infty}{\approx}\left(\int_{-\infty}^{\infty} \cos (M \tau) \sqrt{\frac{\chi}{2 \pi}} \exp \left(-\frac{\chi}{2} \tau^{2}\right) d \tau\right) \cos (M \Delta \omega T) \\
& =\exp \left(-\frac{M^{2}}{2 \chi}\right) \cos (M \Delta \omega T)
\end{aligned}
$$

Since $|\Delta \omega| \ll 2 \pi /(M \cdot T)$ it follows that $\cos (M \Delta \omega T) \approx 1$, and the degradation in (11)-(12) due to carrier frequency error is negligible. Thus for simplicity we set $\Delta \omega=0$, though (11)-(12) provide an easy way to model small frequency errors. Eq. (12) is quite useful because it allows the designer to predict the value of $\hat{l}_{M, N}^{D}$ rather accurately by computing a single exponential.

It is noted that, using Fourier analysis, exact closed-form expressions for $f_{M}^{D}(\chi)$ can be found. This is done in the Appendix, and the expressions are given in (25)-(27).

Plots of (11), (12), and simulated results for $\Delta \omega=0$ are given in Fig. 3; we see that (12) is an excellent approximation. The simulations in Fig. 3 which include quantization effects are quite realistic since they model the following AGC effects: (a) sampler input signal-level backoff (samplers are assumed to be driven at an RMS (Root-MeanSquare) of $80 \%$ of the samplers' full-scale voltage range) and (b) clamping by the samplers when they are saturated. Hence, the simulations presented should be a good prediction of achievable results. If we assume for example $b_{5}=8$ (which would imply an 8bit SNR measurement ${ }^{1}$ in $\mathrm{dB}$ ), then from (4) we have for the simulated quantized systems in Fig. 3 that nbits $=14336$, 30720, 124928, respectively, all of which are very reasonable considering the amount of dedicated memory available in FPGAs (e.g. the various Xilinx Virtex families (see www.xilinx.com)) or which can be implemented in ASICs. Fig. 3 shows that for low $M$ only coarse quantization is needed, while (as expected) higher $M \mathrm{~s}$ require finer quantization to achieve good agreement with the predicted value of $\hat{l}_{M, N}^{D}$. The hardware implementation will be discussed further in Sec. VIII.

B. Conditional Expectation of $\hat{l}_{M, N}^{D}$ given $\bar{\chi}$ for $2 N T \gg T_{C O H}$ The expectation of $\hat{l}_{M, N}^{D}$ conditioned upon $\bar{\chi}$ is:

\footnotetext{
${ }^{1}$ Note that such a measurement could include digits after the binary point. For example, if we put the binary point to the left of the Least Significant Bit (LSB), then we have for an 8-bit output the following: 1 sign bit followed by 6 whole-number bits and 1 fractional bit, which would allow, in 2's complement notation, the representation of the interval $-64 \mathrm{~dB}$ to $+63 \mathrm{~dB}$ in $0.5 \mathrm{~dB}$ intervals, which is usually quite sufficient range and quantization.
} 


$$
\begin{aligned}
& \bar{f}_{M}^{D}(\bar{\chi}) \triangleq E\left[\hat{l}_{M, N}^{D} \mid E\left[\frac{E_{S}}{N_{0}}\right]=\bar{\chi}\right]=\int_{0}^{\infty} f_{M}^{D}(\chi) p_{F}(\chi \mid \bar{\chi}) d \chi \\
& =\cos (M \Delta \omega T) \int_{0}^{\infty}\left(\int_{-2 \pi}^{2 \pi} \cos (M \tau) p_{D}(\tau \mid \chi) d \tau\right) p_{F}(\chi \mid \bar{\chi}) d \chi
\end{aligned}
$$

Due to the infinite number of possible fading distributions, we obviously cannot present results (13) for all fading types. Rather, we shall investigate its behavior under Nakagami- $m$ fading, which is a fading statistic commonly found in systems which use D-MPSK. We again assume $\Delta \omega \approx 0$ and plot theoretical and simulated results for (13) in Fig. 4 for various types of Nakagami- $m$ statistics (note that the "No Fading" curves also correspond to case (a), as verified by comparing Fig. 4 to Fig. 3). The theoretical results were derived using the procedure outlined in the Appendix. Comparing Fig. 4 to Fig. 3 we see that the effect of fading upon the curve of $\hat{l}_{M, N}^{D}$ is rather mild. To evaluate the effects of the fading pdf upon the quantization requirements, we can plot (13) for the various quantizations used in Fig. 3. Such graphs have been omitted due to space constraints, though it is stated that there is no appreciable impact of fading upon the quantization and, hence, upon the hardware resources needed.

\section{C. $\quad$ ariance of $\hat{l}_{M, N}^{D}$}

It can be shown that for $|\Delta \omega| \ll 2 \pi /(M \cdot T)$ and for slow fading the cross-correlation coefficients of $\left\{x_{M, n}^{D}\right\}_{n=-\infty}^{\infty}$ defined as $\rho_{n, k} \triangleq \frac{E\left[x_{M, n}^{D} x_{M, k}^{D}\right]-E\left[x_{M, n}^{D}\right] E\left[x_{M, k}^{D}\right]}{\sqrt{\operatorname{var}\left(x_{M, n}^{D}\right) \operatorname{var}\left(x_{M, k}^{D}\right)}}$ satisfy

$\rho_{n, k}=\left\{1\right.$ for $n=k, \rho_{1}(\chi)$ if $|n-k|=1,0$ if $\left.|n-k|>1\right\}$,

where $\left|\rho_{1}(\chi)\right| \leq 0.3$. Moreover, we can still use [3 Sec. III-D] to surmise $\quad$ that $\quad \forall n, \sigma_{x}^{2} \triangleq \operatorname{var}\left(x_{M, n}^{D}\right) \leq 1$. Now,

$\operatorname{var}\left(\hat{l}_{M, N}^{D}\right)=\operatorname{var}\left(\frac{1}{2 N} \sum_{n=-N+1}^{N} x_{M, n}^{D}\right)$ $=\frac{1}{4 N^{2}}\left(2 N \sigma_{x}^{2}+2 \cdot(2 N-1) \rho_{1}(\chi) \sigma_{x}^{2}\right)$, and using $\sigma_{x}^{2} \leq 1$ and $\left|\rho_{1}(\chi)\right| \leq 0.3$ we have $\operatorname{var}\left(\hat{l}_{M, N}^{D}\right) \leq \frac{1}{4 N^{2}}(2 N+2 \cdot(2 N-1) 0.3) \stackrel{\text { large } \mathrm{N}}{\longrightarrow} \frac{1.6}{2 N}$

Finally, from the central limit theorem for m-dependent variables [12 Theorem 7.3.1, p. 196] we have that $\hat{l}_{M, N}^{D}$ is Gaussian.

\section{Summary: Conditional Distribution of $\hat{l}_{M, N}^{D}$}

Let us unite what we have learned in the previous subsections. We have :

- $\quad$ For case (a): $\hat{l}_{M, N}^{D} \sim N\left(f_{M}^{D}(\chi), \sigma^{2}\right)$ where $f_{M}^{D}(\chi)$ is given in (11) and $\sigma^{2} \leq 1.6 /(2 N)$

- $\quad$ For case (b): $\hat{l}_{M, N}^{D} \sim N\left(\bar{f}_{M}^{D}(\bar{\chi}), \sigma^{2}\right)$ where $\bar{f}_{M}^{D}(\bar{\chi})$ is given in (13) and $\sigma^{2} \leq 1.6 /(2 N)$.

\section{SNR ESTIMATION FROM $\hat{l}_{M, N}^{D}$}

As noted in Section III.B, for case (a) the instantaneous SNR is estimated through (2), while for case (b) the average SNR is estimated through (3). Graphs of $\gamma_{d B}^{D}=10 \cdot \log _{10}\left(\left(f_{M}^{D}\right)^{-1}\left(\hat{l}_{M, N}^{D}\right)\right)$ and $\bar{\gamma}_{d B}^{D}=10 \cdot \log _{10}\left(\left(\bar{f}_{M}^{D}\right)^{-1}\left(\hat{l}_{M, N}^{D}\right)\right)$ are shown in Fig. 5. These curves are the value of LUT \#4 in Fig. 1, and the curve to use would be chosen according to the fading characteristics of the channel. There is an additional small point that needs to be addressed: theoretically we can encounter negative values of $\hat{l}_{M, N}^{D}$, in which case $\bar{\gamma}_{d B}^{D}$ and $\gamma_{d B}^{D}$ would be undefined. This is solved by setting the output ${ }^{2}$ of LUT \#4 to $-2^{b_{5}-1}$ for $\hat{l}_{M, N}^{D} \leq 0$ (not shown in Fig. 5). This correctly reflects the SNR estimate for $\hat{l}_{M, N}^{D} \leq 0$ (which should be $-\infty \mathrm{dB}$, i.e. no signal) within the limits of the available quantization.

There is a very strong relationship between Fig. 5 and Fig. 4. To see this, recall that to graph the inverse of any function, all one has to do is reflect the graph over the line $y=x$. Thus, if we reflect the curves of Fig. 4 over the line $y=x$ then we arrive at Fig. 5.

To quantitatively measure the efficacy of the SNR estimator, following [4 Sec. III] we ask: What is the minimal value of estimation symbol intervals (which is $2 \cdot N$ ) needed to ensure $P\left(\left|\gamma_{d B}^{D}-\chi_{d B}\right|<t o l\right)>C \quad$ (for case (a)) or $P\left(\left|\bar{\gamma}_{d B}^{D}-\bar{\chi}_{d B}\right|<t o l\right)>C$ (for case (b)) where tol is the tolerance and $C$ is the confidence. For example, some appropriate values of tol and $C$ would be $t o l=1.5 \mathrm{~dB}$ and $C=95 \%$. Straightforward following of the development of [4 eq. (15)] shows that the answer is:

$$
2 N>2 \cdot 1.6 \cdot \frac{\left(\operatorname{erf}^{-1}(C)\right)^{2}}{\left.\min \left\{\begin{array}{l}
\left|\ell\left(\left(1-w_{2}\right) \cdot \alpha\right)-\ell(\alpha)\right|, \\
\left|\ell\left(\left(1+w_{1}\right) \cdot \alpha\right)-\ell(\alpha)\right|
\end{array}\right\}\right)^{2}}
$$

where $w_{1} \triangleq\left(10^{\text {tol } / 10}-1\right)$ and $w_{2} \triangleq\left(1-10^{-t o l / 10}\right)$, and: for case (a): $\ell(\bullet) \triangleq f_{M}^{D}(\bullet)$ and $\alpha \triangleq \chi$, and for case (b): $\ell(\bullet) \triangleq \bar{f}_{M}^{D}(\bullet)$ and $\alpha \triangleq \bar{\chi}$.

\section{DEVELOPMENT OF A COMPARISON YARDSTICK}

Following the discussion in [4 Sec. IV] we note that many demodulators generate SNR estimates by measuring the pre- or postdecoder error rate. For example, this is done in systems that estimate the SNR from the number of errors detected in preambles, pilot symbols, or "training sequences" that are embedded in the data stream. Therefore, as noted in [4 Sec. IV], perhaps the most meaningful and universally applicable yardstick by which to measure the efficacy of SNR estimation via $\hat{l}_{M, N}^{D}$ is through comparison of (15) to the number of symbols needed for SNR estimation through measurement of the pre-decoder Symbol Error Rate (SER).

SNR estimation from the SER is based upon the principle that the

\footnotetext{
${ }^{2}$ This is the value of the LUT output if the representation is of whole numbers (not necessarily the case, see Footnote 1). Generally, the idea is to use the lowest SNR expressible via the LUT's quantization.
} 
SER is always a strictly monotonically decreasing function of the SNR (in words: the higher the SNR, the lower the SER). Suppose we denote the SER function as $h(\chi)$ or $h(\bar{\chi})$, then if we measure the SER upon $L$ received symbols, and denote this measured SER as $S(L)$, then an estimate of the SNR may be obtained via $h^{-1}(S(L))$. See [4 Sec. IV] for a more thorough discussion of this point.

In this paper (unlike in [4]) we are also addressing the effects of fading, so we must differentiate between two cases: case (i): $L \cdot T \ll T_{\mathrm{COH}}$, and case (ii): $L \cdot T \gg T_{\mathrm{COH}}$. The symbol error rate for D-MPSK for case (i) is [13 eq. (3)]:

$g_{M}^{D}(\chi) \triangleq \frac{1}{\pi} \int_{0}^{\pi-\pi / M} \exp \left(-\chi \cdot \frac{\sin ^{2}(\pi / M)}{1+\cos (\pi / M) \cos \xi}\right) d \xi$

The SER for D-MPSK when $L \cdot T \gg T_{\mathrm{COH}}$ is accordingly:

$\bar{g}_{M}^{D}(\bar{\chi}) \triangleq \int_{0}^{\infty} g_{M}^{D}(\chi) p_{F}(\chi \mid \bar{\chi}) d \chi$

SNR estimation from the SER is done analogously to (2) and (3), namely, for case (i) we can estimate the instantaneous SNR via:

$\eta_{d B}^{D}=10 \cdot \log _{10}\left(\left(g_{M}^{D}\right)^{-1}(S(L))\right)$

while for case (ii) we estimate the average SNR through:

$\bar{\eta}_{d B}^{D}=10 \cdot \log _{10}\left(\left(\bar{g}_{M}^{D}\right)^{-1}(S(L))\right)$

For judging the efficacy of estimation via the SER, we ask: What is the minimal value of $L$ needed to ensure $P\left(\left|\eta_{d B}^{D}-\chi_{d B}\right|<t o l\right)>C \quad$ (for case (i)) or $P\left(\left|\bar{\eta}_{d B}^{D}-\bar{\chi}_{d B}\right|<t o l\right)>C$ (for case (ii)). From a derivation similar to that which led to [4 eq. (22)], we find that the answer to this question is:

$$
L>\frac{2 h(\beta)(1-h(\beta))\left(\operatorname{erf}^{-1}(C)\right)^{2}}{\left.\min \left\{\begin{array}{l}
\left|h\left(\left(1-w_{2}\right) \cdot \beta\right)-h(\beta)\right|, \\
\left|h\left(\left(1+w_{1}\right) \cdot \beta\right)-h(\beta)\right|
\end{array}\right\}\right)^{2}}
$$

where for case (i): $h(\bullet) \triangleq g_{M}^{D}(\bullet)$ and $\beta \triangleq \chi$, and for case (ii): $h(\bullet) \triangleq \bar{g}_{M}^{D}(\bullet)$ and $\beta \triangleq \bar{\chi}$.

\section{GRAPHICAL EXHIBITION OF RESULTS}

Graphs of (15) vs. (20) for cases (a) and (i) are given in Fig. 6, where we see that the proposed estimator often requires considerably fewer symbol intervals in order to arrive at an equally accurate estimate. The lowest SNRs for which results are given in Fig. 6 are rough thresholds $\Gamma_{M}$ defined as the SNRs at which $g_{M}^{D}\left(\Gamma_{M}\right) \approx 5 \cdot 10^{-2}$. This SER was chosen as the threshold because only at SNRs above those which produce such a pre-decoder SER is it likely that use of coding algorithms would reduce the error rate enough in order to render the received data useful.

In Fig. 7 we present results ${ }^{3}$ for case (b) and (ii), for $M=4$

\footnotetext{
${ }^{3}$ We presented here graphs of case (a) and case (i) (in Fig. 6) and case (b) and (ii) (in Fig. 7). Theoretically, there could be situations where $L$ and $N$ are such that one would have to compare case (a) to case (ii) or case (b) to case (i). Such a comparison can be made by looking at the appropriate curves taken from Fig. 6 and Fig. 7, or similar figures.
}

(QPSK) with Nakagami- $m$ fading for various values of $m$ (results for $M=2$ and $M=8$ show a similar behavior, and are hence omitted due to space constraints). As can be seen in that figure, the advantage of the proposed technique is more pronounced for higher $m$ 's. As $m$ increases the performance approaches that of case (a) vs. case (i), as shown in Fig. 6. This is explained by the fact that as $m \rightarrow \infty$ the Nakagami- $m$ fading behavior approximates a no-fading situation [1 Sec. 14.3]. Again, the lowest SNRs for which results are given in Fig. 7 are rough thresholds $\bar{\Gamma}_{M}$ defined as the average SNRs at which $\bar{g}_{M}^{D}\left(\bar{\Gamma}_{M}\right) \approx 5 \cdot 10^{-2}$. Note that for Nakagami- $m$ fading we have [14 eqs. (3), (13)]:

$$
\bar{g}_{M}^{D}(\bar{\chi}) \triangleq \frac{1}{\pi} \int_{0}^{\pi-\pi / M}\left(1+\frac{\sin ^{2}(\pi / M)}{1+\cos (\pi / M) \cos \xi} \cdot \frac{\bar{\chi}}{m}\right)^{-m} d \xi
$$

\section{DISCUSSION AND CONCLUSIONS}

The analysis of the results presented in Fig. 6 to Fig. 7 will proceed in a manner similar to that of [4 Sec. V], though because in [4] fading effects were not treated, here we engage in a slightly more complicated discussion. By inspection of Fig. 6 and Fig. 7, the number of symbols needed for the estimation of the SNR from $\hat{l}_{M, N}^{D}$ does not change significantly as a function of the fading characteristics and coherence time. In contrast, estimation via the SER is strongly affected by the fading characteristics and the coherence time.

Let us first treat operation at high SNR. At high SNR we observe that estimation via $\hat{l}_{M, N}^{D}$ in general requires much less symbol intervals than estimation via the SER. This means that the proposed estimator can generate estimates much more rapidly than estimation via the SER. Fig. 7 shows that, for case (b), as the fading index $m$ increases, so does the advantage of the proposed estimator. For moderate and high $m$, and, as well, for case (a) ( $2 N T \ll T_{\mathrm{COH}}$, shown in Fig. 6) we find that the proposed estimation method is much better than estimation via the SER, often by many orders of magnitude.

Now let us discuss low SNR operation. At low SNRs, we see that the proposed method requires about the same number of symbols as SNR estimation via the SER. Since it is often the case that the receiver spends most of its lifetime operating in the low-SNR region, one could make the argument that the advantage of the proposed method is minimal since it requires about the same number of symbol intervals as estimation via the SER. This, however, ignores several key issues. First, consider the case of unknown data being transmitted. The only way by which an SER estimate can be obtained from unknown data is by obtaining an error rate estimate from a code-decode process [15]. This means that one must first code the transmitted data at the transmitter and then decode it at the receiver (e.g., using block codes or convolution codes), and that in order to obtain an error rate estimate the receiver would compare the decoded data stream to the input data stream, hence arriving at an error rate estimate. This, however, implicitly assumes that the error correction decoder (ECD) output is completely error free - which is a fallacy at low SNRs. Hence, at lower SNRs the SER estimate would be inherently unreliable, with this problem being more severe as the SNR decreases. Moreover, the ECD may not even be locked at low SNRs, hence precluding SER estimation. To combat this problem, known symbols can be sent over the channel (in the form of training sequences, pilot symbols, or preambles) and the error rate estimation can be done upon those symbols. This, however, introduces two problems. First, obviously, the channel throughput that is taken up by those symbols cannot be 
used in order to transmit data, i.e. a reduction in the channel's information throughput is incurred. Secondly, unless we are prepared to significantly shut down the information-bearing content of the channel, the known symbols must only be allowed to take up a small percentage of the data stream. If we call this percentage $P$ (e.g., $P=10 \%$ ), then we have that the number of symbol intervals that we actually have to wait in order to arrive at the SER estimate is increased by a factor of $1 / P$ over the quantities outlined in Fig. 6 and Fig. 7. For example, for $P=10 \%$ we would need to multiply those quantities by a factor of 10 , which clearly degrades the performance as compared to estimation via $\hat{l}_{M, N}^{D}$. Therefore, we can say that the results in Fig. 6 and Fig. 7 are optimistic with regards to estimation via the SER, and that, consequently, the proposed method is also superior at low SNRs.

In terms of complexity, we note that estimation via the SER requires the implementation of error detection and accrual mechanisms, which often necessitate a non-trivial amount of hardware and/or software resources. This, in addition to an algorithm or lookup table to translate the SER measurement into an SNR estimate. In contrast, the proposed method is impervious to the content of the data stream, the coding method, and the error rate. Regarding fixed-point implementation of the proposed estimator, we make note of the fact that the value of tol must, obviously, be larger than the minimum resolution achievable given the quantization. In Sec. VII we assumed that enough quantization bits were used and we ignore quantization effects (a good assumption, considering Sec. IV). Moreover, it is easily shown that accurate fixed-point hardware estimation of the SNR from the SER would require an unfeasibly large LUT (due to the large dynamic range of the SER). Thus, including quantization effects would have heavily favored estimation via $\hat{l}_{M, N}^{D}$ even more.

Let us now delve even further into hardware complexity analysis. As noted in Section III.A, in D-MPSK systems detection of the received symbols is often achieved via generating a pseudo-coherently demodulated M-PSK signal $u_{n}$ (see [1 Sec. 5.2.8]). However, an equally valid detector would be via generation of the normalized pseudo-coherently demodulated M-PSK signal $v_{n}$. In fact, it is trivial to see that the latter has advantages in terms of the stability its dynamic range vis-à-vis the AGC's operation. Thus, implementation of $v_{n}$ in Fig. 1 obviates the need to generate the constellation $u_{n} \triangleq r_{n} r_{n-1}^{*}$, and, hence, it can be argued that the only real hardware penalty incurred by implementing the proposed estimator is the sequence LUT\#3 $\rightarrow$ IAD $\rightarrow$ LUT\#4 (see Fig. 1), which is the same order of complexity as the Linn-Peleg estimator ([3], [4]), i.e., trivial.

In this paper we decided to focus on comparisons of the proposed method versus estimation via the SER. Other SNR estimators have been suggested in [15], [16], and [17]. In theory, these could be applied to the pseudo-coherent constellation $v_{n}$ in order to produce an SNR estimate. While, due to space constraints, in-depth comparison vs. those estimators is impossible within the span of the current paper, we shall claim that the proposed estimator possesses several qualitative advantages which would indicate a favorable outcome to such a comparison. To this end, it shall be commented that of the estimators presented in [15]-[17]: • some will work only within certain receiver structures, $\cdot$ most require some form of symbol decisions to be made, - most need more than one sample/symbol, and (most importantly) - none of those methods seem to have a hardware implementation nearly as compact as the one suggested in this paper.

As additional observations, we note that due to the fact that the current estimator is based upon usage of the normalized pseudo- coherent constellation applied to the Linn-Peleg estimator ([3], [4]), the proposed estimator retains the resilience to AGC circuit imperfections of the latter (see [3], [4]). Due to this same genealogy, a very important observation is that the proposed SNR estimator can be used to provide an SNR estimate for coherent M-PSK but without the need for carrier synchronization (which is a prerequisite for the LinnPeleg estimator). We note, however, that in general SNR estimation for coherent M-PSK via $\hat{l}_{M, N}^{D}$ requires more symbol intervals than the Linn-Peleg estimator, and, thus, the latter should be used when carrier synchronization has been achieved.

Finally, it is mentioned that it can be shown through Fourier analysis that $f_{M}^{D}(\chi)=\left(f_{M}(\chi)\right)^{2}$ (where $f_{M}(\chi)$ $\triangleq E\left[\hat{l}_{M, N} \mid E_{S} / N_{0}=\chi\right]$ (see [4])) and, furthermore, the techniques of this paper can conceivably be generalized for SNR estimation for $\mathrm{D}^{\mathrm{n}} \mathrm{PSK}$ modulations (defined in [10 Chap. 8.1]), an issue that is currently being explored by the author. However, these issues are beyond the scope of this paper.

\section{APPENDIX - EXACT CLOSED-FORM EXPRESSIONS FOR $f_{M}^{D}(\chi)$}

Through use of Fourier analysis, closed-form expressions may be attained for $f_{M}^{D}(\chi)$. To do so, we first re-define for convenience the density function $p_{D}\left(\Delta \phi^{D} \mid \chi\right)$ over the interval $[-\pi, \pi]$ (this is possible because the true phase is in the interval $[-\pi, \pi]$, as noted in Section IV). We name this distribution $\tilde{p}_{D}\left(\Delta \tilde{\phi}^{D} \mid \chi\right)$ (where $\left.\Delta \tilde{\phi}^{D} \in[-\pi, \pi]\right)$, and it is given by [10 eq. (7.3), p. 441]: $\tilde{p}_{D}\left(\Delta \tilde{\phi}^{D} \mid \chi\right)$

$=\frac{1}{2 \pi} \int_{0}^{\pi / 2}(\sin \tau) \cdot\left(1+\chi\left(1+\cos \Delta \tilde{\phi}^{D} \sin \tau\right)\right) e^{-\chi\left(1-\cos \Delta \tilde{\phi}^{D} \sin \tau\right)} d \tau$

With this definition of $\tilde{p}_{D}$ we find from (11) that (assuming for simplicity $\Delta \omega=0$ ):

$f_{M}^{D}(\chi)=\int_{-\pi}^{\pi} \cos \left(M \cdot \Delta \tilde{\phi}^{D}\right) \tilde{p}_{D}\left(\Delta \tilde{\phi}^{D} \mid \chi\right) d\left(\Delta \tilde{\phi}^{D}\right)$

(Note that the limits of the integral in $(23)$ are $[-\pi, \pi])$. Now, because the domain of $\tilde{p}_{D}$ is finite, the periodic extension of $\tilde{p}_{D}$ can be expressed as a Fourier series, i.e. $\tilde{p}_{D}\left(\Delta \tilde{\phi}^{D} \mid \chi\right)=\frac{1}{2 \pi} \sum_{m=-\infty}^{\infty} c_{m}^{D}(\chi) \exp \left(-j m \Delta \tilde{\phi}^{D}\right)$ where, using the fact that $\tilde{p}_{D}$ is even:

$c_{m}^{D}(\chi)=\int_{-\pi}^{\pi} \cos \left(m \cdot \Delta \tilde{\phi}^{D}\right) \tilde{p}_{D}\left(\Delta \tilde{\phi}^{D} \mid \chi\right) d\left(\Delta \tilde{\phi}^{D}\right)$

(see [18 App. 4A]). From comparing (24) to (23), we see that $f_{M}^{D}(\chi)=\left.c_{m}^{D}(\chi)\right|_{m=M}$. The coefficients $c_{m}^{D}(\chi)$ were computed in [18 App. 4A], from which it follows that (see [18 eq. (4.A.18)]):

$f_{M}^{D}(\chi)=\left.c_{m}^{D}(\chi)\right|_{m=M}=\frac{\pi \chi}{4} \cdot e^{-\chi}\left[I_{\frac{M-1}{2}}\left(\frac{\chi}{2}\right)+\frac{I_{\frac{M+1}{2}}}{2}\left(\frac{\chi}{2}\right)\right]^{2}$

where $I_{k}(\bullet)$ is the $k$-th order modified Bessel function of the first kind (see [11 Chap. 24]). We can simplify (25) even further when we 
note that since $M$ always an even number, we have that $\frac{M-1}{2}$ and $\frac{M+1}{2}$ are half an odd integer, and then there are closed-form expressions for $I_{(M-1) / 2}(\bullet)$ and $I_{(M+1) / 2}(\bullet)$ in terms of hyperbolic functions [11 eqs. (24.46), (24.58)-(24.63)], which can be simplified even further by decomposing the hyperbolic functions into exponentials [11 eq. (8.1), (8.2)]. Following this procedure, we arrive at the following expressions:

$$
\begin{aligned}
& f_{2}^{D}(\chi)=1-2 \chi^{-1}+\chi^{-2}+2 \chi^{-1} e^{-\chi}-2 \chi^{-2} e^{-\chi}+\chi^{-2} e^{-2 \chi} \\
& f_{4}^{D}(\chi)=1-8 \chi^{-1}+28 \chi^{-2}-48 \chi^{-3}+36 \chi^{-4}-4 \chi^{-1} e^{-\chi}+4 \chi^{-2} e^{-\chi} \\
& +4 \chi^{-2} e^{-2 \chi}+24 \chi^{-3} e^{-\chi}+24 \chi^{-3} e^{-2 \chi}-72 \chi^{-4} e^{-\chi}+36 \chi^{-4} e^{-2 \chi}
\end{aligned}
$$

Expressions for $M>4$ can also be found. However, since those expressions are very tedious and since they can be arrived at following the same procedure outlined above, they are omitted due to space constraints. Moreover, we note that the approximation (12) is extremely accurate for $M>2$ (see Fig. 3).

Another application of (26)-(27) is in the computation of $\bar{f}_{M}^{D}(\bar{\chi})$ via (13). For certain fading distributions, this can lead to closed form expressions for $\bar{f}_{M}^{D}(\bar{\chi})$. Due to space constraints such results are omitted here. However, it shall be noted that exact (though very long) closed-form expressions for $\bar{f}_{M}^{D}(\bar{\chi})$ can be obtained for Nakagami- $m$ fading for all $M$ and all $m$ through computation of the definite integral $\bar{f}_{M}^{D}(\bar{\chi})=\int_{0}^{\infty} f_{M}^{D}(\chi) \frac{m^{m} \chi^{m-1}}{\bar{\chi}^{m} \Gamma(m)} \exp \left(\frac{-m \chi}{\bar{\chi}}\right) d \chi \quad$ by using (26)-(27) (or similar expressions for $M>4$ ), along with the formula for the definite integral $\int_{0}^{\infty} y^{n} \exp (-a y) d y=\Gamma(n+1) / a^{n+1} \quad([11$ eq. (15.76)]). In fact, this has been done by the author and those are the theoretical predictions that have been plotted in Fig. 4 (and which are completely validated by the simulation results). As a side note it is commented that if the reader is to attempt such a computation, it is recommended that if $m$ is an integer that a value $\tilde{m}=m+\varepsilon$ be used instead of $m$, where $0<\varepsilon \ll m$ (e.g. $\varepsilon=m / 1000$ ). This will solve the problem of some singularities caused during the integration.

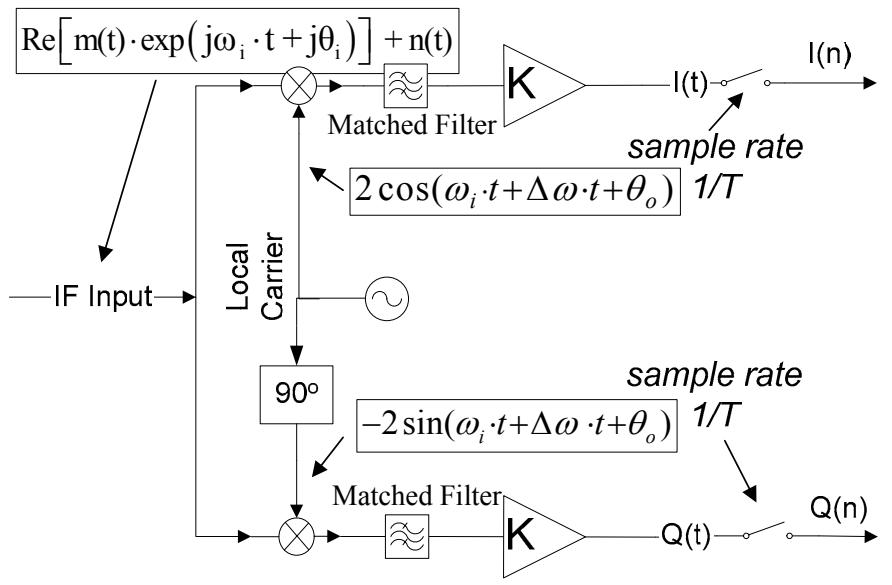

Fig. 1 - Front end of D-MPSK receiver (simplified diagram).

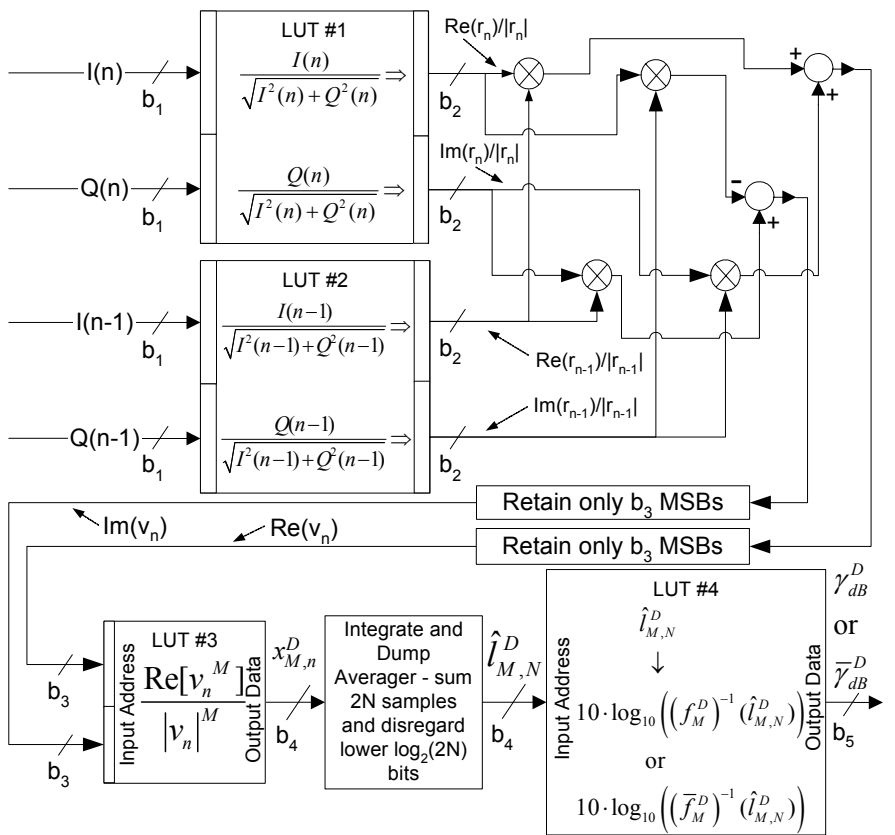

Fig. 2 - Fixed-point hardware generation of $\gamma_{d B}^{D}$ or $\bar{\gamma}_{d B}^{D}$.

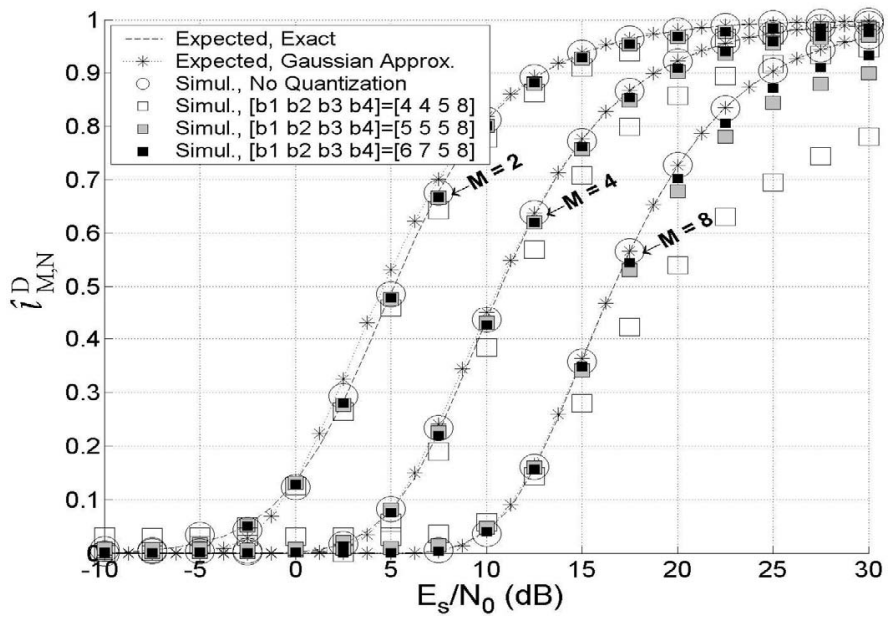

Fig. $3-\hat{l}_{M, N}^{D}$ for $2 N T \ll T_{C O H}$. Note the similar shape (but very different values) vs. [3 Fig. 3].

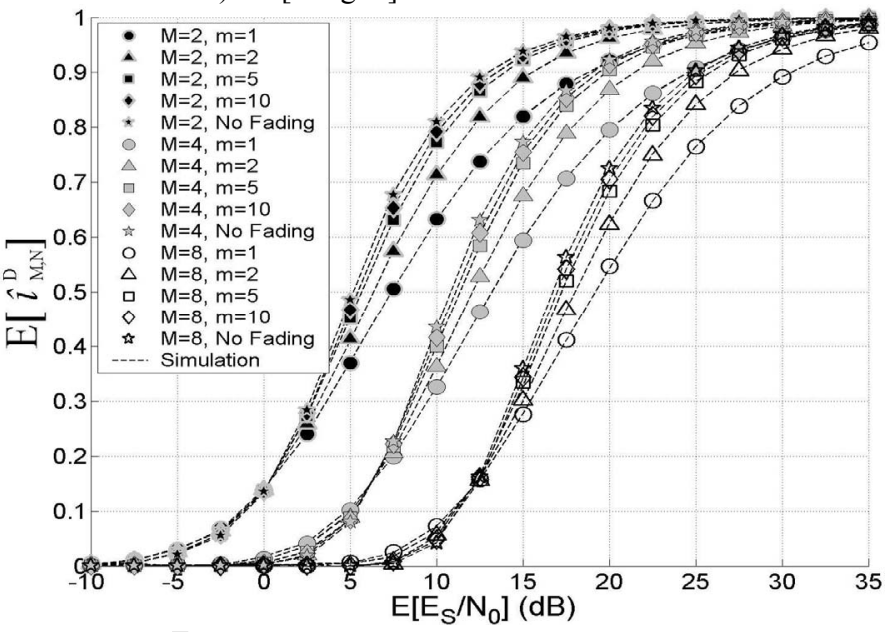

Fig. $4-\bar{f}_{M}^{D}(\bar{\chi})$ vs. $\bar{\chi}$ for Nakagami- $m$ fading: theory and simulations. Quantization effects are ignored. 


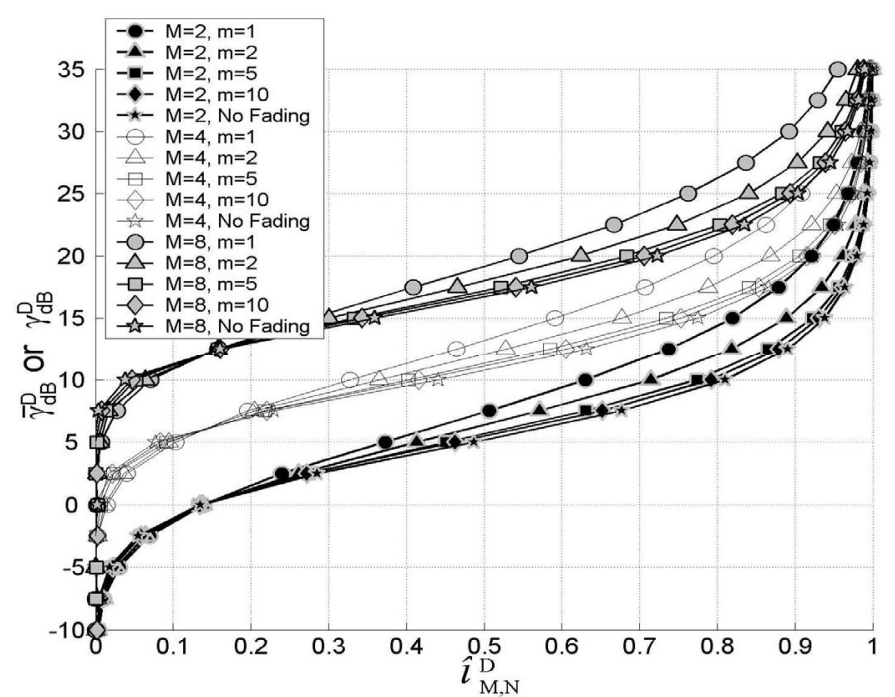

Fig. $5-\gamma_{d B}^{D}$ (for case (a); these are the "No Fading" curves, see Sec. IV.A) or $\bar{\gamma}_{d B}^{D}$ (for case (b)) vs. $\hat{l}_{M, N}^{D}$.

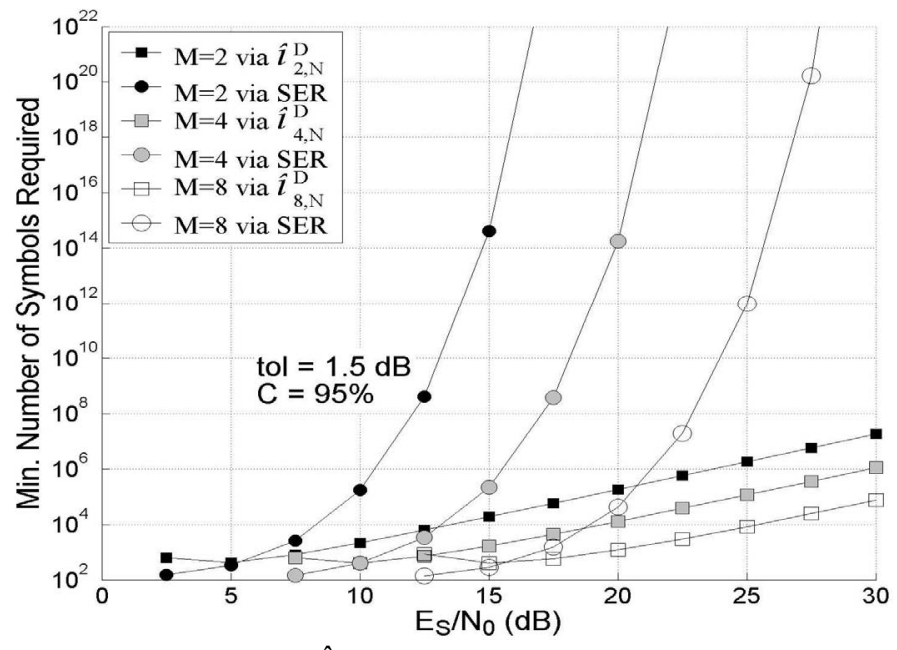

Fig. 6 - Estimation via $\hat{l}_{M, N}^{D}$ vs. estimation via the SER for cases (a) and (i).

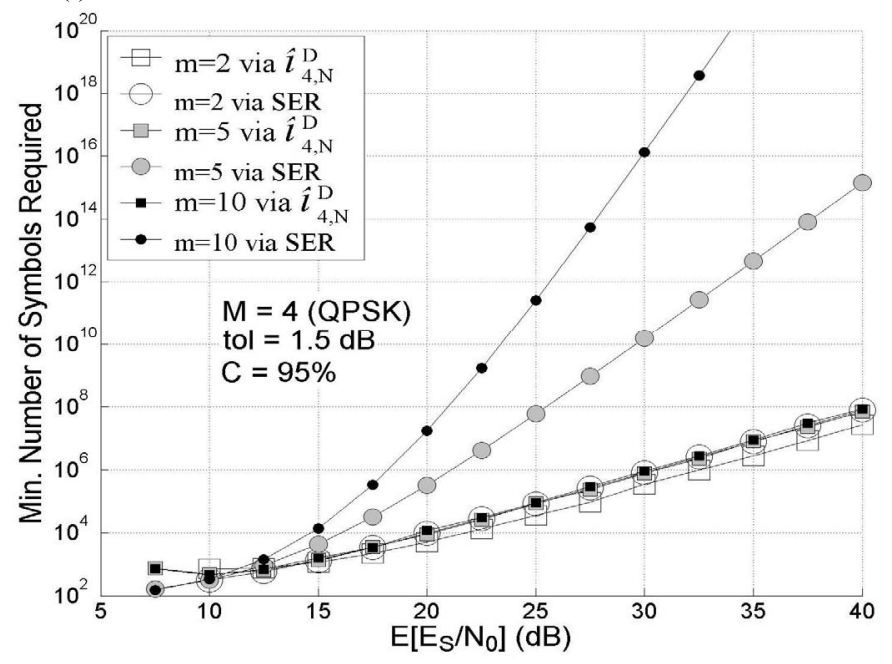

Fig. 7 - Estimation via $\hat{l}_{M, N}^{D}$ vs. estimation via the SER for cases (b) and (ii). Modulation is QPSK, and Nakagami- $m$ fading is present.

\section{ACKNOWLEDGMENT}

The author gratefully acknowledges the financial support given to him by NSERC (National Sciences and Engineering Research Council of Canada) and Bell Canada, the logistical support of Prof. Matthew J. Yedlin (University of British Columbia), and the kind assistance given by the Sarnoff Conference Committee, in particular by Prof. Nirwan Ansari (New Jersey Institute of Technology).

\section{REFERENCES}

[1] J. G. Proakis, Digital communications, 4th ed. Boston: McGrawHill, 2001.

[2] T. A. Summers and S. G. Wilson, "SNR mismatch and online estimation in turbo decoding," IEEE Trans. Commun., vol. 46, no. 4, pp. 421-423, Apr. 1998.

[3] Y. Linn and N. Peleg, "A family of self-normalizing carrier lock detectors and Es/N0 estimators for M-PSK and other phase modulation schemes," IEEE Trans. Wireless Commun., vol. 3, no. 5, pp. 1659-1668, Sep. 2004.

[4] Y. Linn, "Quantitative analysis of a new method for real-time generation of SNR estimates for digital phase modulation signals," IEEE Trans. Wireless Commun., vol. 3, no. 6, pp. 19841988, Nov. 2004.

[5] Y. Linn, "A Robust Phase Detection Structure for M-PSK: Theoretical Derivations, Simulation Results, and System Identification Analysis," in Proc. 18th Canadian Conference on Electrical and Computer Engineering (CCECE'05), Saskatoon, Saskatchewan, Canada, May 1-4, 2005, pp. 869-883.

[6] S. Haykin, Communication systems, 2nd ed. NY: Wiley, 1983.

[7] M. K. Simon and M. Alouini, "A unified approach to the performance analysis of digital communication over generalized fading channels," Proc. IEEE, vol. 86, no. 9, pp. 1860-1877, Sep. 1998.

[8] R. F. Pawula, S. O. Rice, and J. H. Roberts, "Distribution of the phase angle between two vectors perturbed by Gaussian noise," IEEE Trans. Commun., vol. 30, no. 8, pp. 1828-1841, Aug. 1982.

[9] R. Pawula, "On M-ary DPSK Transmission Over Terrestrial and Satellite Channels," IEEE Trans. Commun., vol. 32, no. 7, pp. 752-761, Jul. 1984.

[10] M. K. Simon, S. M. Hinedi, and W. C. Lindsey, Digital communication techniques. NJ: Prentice Hall, 1995.

[11] M. R. Spiegel, Mathematical handbook of formulas and tables. NY: McGraw-Hill, 1968.

[12] K. L. Chung, A course in probability theory. NY: Harcourt, 1968.

[13] R. F. Pawula, "Generic error probabilities," IEEE Trans. Commun., vol. 47, no. 5, pp. 697-702, May 1999.

[14] S. Hyundong and L. Jae Hong, "On the error probability of binary and M-ary signals in Nakagami-m fading channels," IEEE Trans. Commun., vol. 52, no. 4, pp. 536-539, Apr. 2004.

[15] N. Celandroni, E. Ferro, and F. Potorti, "Quality estimation of PSK modulated signals," IEEE Commun. Mag., vol. 35, no. 7, pp. 50-55, Jul. 1997.

[16] D. R. Pauluzzi and N. C. Beaulieu, "A comparison of SNR estimation techniques for the AWGN channel," IEEE Trans. Commun., vol. 48, no. 10, pp. 1681-1691, Oct. 2000.

[17] M. K. Simon and S. Dolinar, "Improving SNR estimation for autonomous receivers," IEEE Trans. Commun., vol. 53, no. 6, pp. 1063-1073, Jun. 2005.

[18] K.-P. Ho, Phase-modulated optical communication systems. NY: Springer, 2005. 
Disclaimer: 1-4244-0002-3/06/\$20.00 @2006 IEEE. 